\title{
Dynamic aperture experiment at a synchrotron
}

\author{
W. Fischer, M. Giovannozzi, and F. Schmidt \\ CERN, CH-1211 Geneva 23, Switzerland \\ (Received 29 November 1995; revised manuscript received 18 September 1996)
}

\begin{abstract}
The dynamic aperture experiment at the CERN Super Proton Synchrotron (SPS) was aimed at finding the relevant effects that limit single-particle stability in hadron storage rings. These effects were studied in the SPS and compared with long-term particle tracking to determine to what extent computer simulations can predict the dynamic aperture under well-known conditions. Such investigations are very important for future hadron colliders such as the Large Hadron Collider as the design of these machines relies heavily on simulations. Besides this practical goal it was of utmost interest to improve the phenomenological understanding of the intricate details of particle motion in phase space. This experiment was carried out by successive teams over a period of ten years. We summarize the techniques, results, and conclusions. [S1063-651X(97)10602-X]

PACS number(s): 29.20.-c
\end{abstract}

\section{INTRODUCTION}

All high-energy hadron accelerators currently planned or under construction need superconducting technology to reach the high fields needed to guide and focus the beams. Whereas in the classic technology the required fields were reproduced by accurately shaping the magnetic pole pieces, in superconducting magnets the field quality depends almost entirely on the position and properties of the superconducting filaments inside the coils. As a result it is more difficult to avoid unwanted multipolar errors that affect the particle dynamics $[1,2]$.

Since hadrons lose a negligible part of their energy through synchrotron radiation, their motion in phase space can be adequately described in the framework of the Hamiltonian formalism. In such conservative systems, the nonlinearities can make the motion of the particles chaotic in parts of the phase space through which they slowly migrate outwards until they are lost at some obstacle in the beam pipe.

It is of utmost importance for the design of a machine like the Large Hadron Collider (LHC), the $7 \mathrm{TeV}$ on $7 \mathrm{TeV}$ proton collider planned in the Large Electron-Positron (LEP) tunnel at CERN, to understand in detail the nonlinear dynamics of the circulating particles. The main purpose of the dynamic aperture experiment at the Super Proton Synchrotron (SPS) was therefore to simulate a nonlinear machine including tune modulation, which is known to enhance the destabilizing effects of nonlinearities [3], and to investigate particle losses under these conditions.

The dynamic aperture, corresponding to the stable phasespace area, can be defined for our purpose as the maximum betatron amplitude below which no particle loss takes place within a time interval of interest. In the case of the LHC, the injection time is the relevant period since the transverse magnetic field errors attain large values and the particles have to stay at injection for $15 \mathrm{~min}$ with the beam size at its maximum. Unfortunately there is at present no tool or technique available to evaluate these loss times despite a decade of intense theoretical research. Hence, we have to rely on bruteforce tracking. One of the main motivations for the experiments was to use the SPS as a test bench to compare experi- mental results from a controlled nonlinear machine with the predictions from tracking.

Answering this important practical problem was not the only incentive for the aperture studies. Much effort has been devoted to improving our phenomenological understanding of the intricate nature of nonlinear motion in phase space. We hope that theorists will find a consistent way to describe the slow-particle-loss mechanisms observed in the SPS experiment.

The next section puts our studies into context with experiments at other accelerator centers and gives a general overview of the results of the SPS experiment up to 1991. Section III describes the instrumentation necessary for preparing the SPS machine. The experimental conditions, the tracking model, and preparatory experiments are stated. In Sec. IV the experiment with scrapers is explained together with experimental and tracking results. The experimental dynamic aperture is compared with tracking results in Sec. V. Finally, in Sec. VI, a detailed summary is given of all issues concerning our improved insight into nonlinear particle motion in hadron storage rings.

\section{HISTORICAL OVERVIEW}

\section{A. Experiments on transverse nonlinear dynamics}

Transverse nonlinear resonance phenomena have been of interest to the accelerator community for many years (see Ref. [4]). With the planning of a new generation of large hadron colliders like the Superconducting Super Collider (SSC) and the Large Hadron Collider (LHC), intense experimental activity was started in the U.S. at Fermilab, and in Europe at the CERN Super Proton Synchrotron (SPS) and the DESY Hadronen Elektronen Ring Anlage (HERA) proton ring (see Refs. [5-7], respectively).

In the Fermilab E778 experiment [8] the measurements were predominantly made in the vicinity of the $2 / 5$ resonance. Various measurements were performed: detuning with amplitude, "smear" measurements, island capture (including tune modulation), and time-dependent beam profiles. The theoretical treatment relied on the Hamiltonian formalism, the phase diagram was studied in depth, and the beam losses were described by a diffusive model. 
In all those experiments sextupole magnets served as the dominant source of nonlinearities with the exception of the HERA experiment. There the dynamic aperture due to the multipolar errors of the superconducting magnets was measured under normal operating conditions. In this experiment a good agreement between the tracking results (all known magnetic errors considered [7]) and the measurements was achieved when the experimental conditions were well understood.

Dynamic aperture experiments have also been performed at the Indiana University Cyclotron Facility (IUCF) in which low-order resonances with and without tune modulation were studied and compared with Hamiltonian models (see Refs. [9] and [10]). At the Aladdin electron ring at the Synchrotron Radiation Center in Stoughton, Wisconsin, third-order resonances were studied [11] as well. The results were similar to those found in conservative systems since experimental periods were studied that were small compared to the damping time. More recently, studies have been started at the SPEAR storage ring at Stanford to analyze the full six-dimensional phase space [12].

\section{B. The SPS experiment: Measurements and simulations}

The purpose of this section is to summarize the SPS experiments up to the end of 1991 . We will restrict ourselves here to a discussion of the results while a thorough description of the experimental conditions will be given in the next section.

The early experiments can be grouped into three periods. In the initial phase [13] in 1986 the basic machine setup was defined and first short-term results were obtained. In 1988 the short-term dynamic aperture was studied in detail and a slow loss process was found [14]. From 1989 to 1991 an attempt was made [15] to understand this loss process quantitatively. To this end the experimental setup, the instrumentation, and the measurement and simulation techniques were revised. Some progress could be reported but reliable results have only been obtained in the period from 1992-1994. They will be discussed later.

\section{Experimental session in 1986}

The operational conditions of the SPS were carefully chosen so as to obtain a well-tunable and very linear machine. With these conditions as a starting point, the SPS was made nonlinear in a controlled way with eight strong sextupoles. Two different configurations were tested: one leads to a strong excitation of the third-order resonance, a low-order resonance that is to be avoided for safe operation of a collider; the other configuration suppresses this resonance so that the particle motion is dominated by higher-order resonances. This has a greater resemblance to a machine such as the LHC, which is very nonlinear due to the strong multipolar errors of its superconducting magnets. The latter configuration has been exclusively used for all following experiments.

Wire scans were used to visualize a kicked beam: after one kick one finds the signature of a hollow beam filamented in phase space that shows a double-peak structure in the projection. A second kick partly restores the original onepeak structure that the beam had before the kick. Moreover,

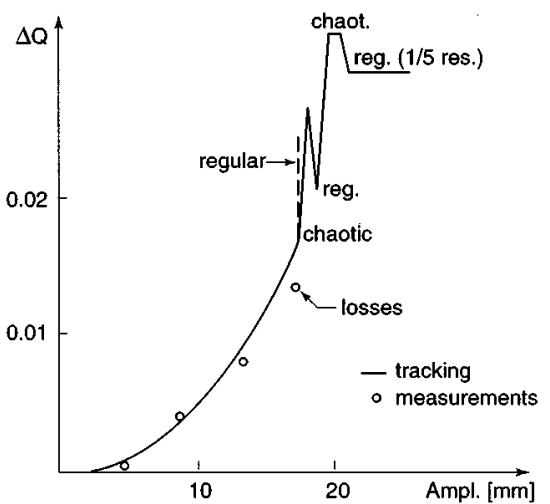

FIG. 1. Comparison of tracking with experiment (close to fifthorder resonance).

close to the third-order resonance the wire-scan profile is distorted revealing phase-space deformations due to this resonance. Another important tool was the Schottky detector, which allows a measurement of the tune distributions with a high resolution, allowing one to detect easily the losses due to particular resonances.

The experiment mainly studied the short-term (few seconds of SPS storage time or some $10^{5}$ turns) dynamic aperture of various working points close to the fifth- and seventhorder resonances. Tracking simulations over $10^{2}-10^{3}$ turns could predict the short-term dynamic aperture quite well, provided the measured closed orbit and synchrotron oscillations were taken into account. The presence of a vertical closed orbit turned out to generate instabilities through excitation of skew resonances. Therefore, in all following experiments, the closed orbit (especially the vertical one) was carefully measured and corrected and the residual orbit was included in the simulations.

Finally, long-term experiments (some minutes of storage time) were started without the synchrotron oscillations to avoid the additional effect due to the too strong RF noise. This configuration was used in all the subsequent experimental studies.

\section{Experimental session in 1988}

In this session two working points were studied close to the nest of coupling resonances of fifth and seventh order, respectively. The detuning with amplitude was measured and found to be in good agreement with tracking. An example is shown in Fig. 1, which also shows the good agreement between the border of chaotic motion, as determined in the tracking, and the amplitude where short-term losses start to be visible in the experiment. As expected, this short-term dynamic aperture is smaller for the fifth-order resonance due to the larger driving terms according to perturbation theory.

Although the basic nonlinear parameters were well under control and the short-term dynamic aperture was well predicted by tracking, a slow particle transport was found in the long-term measurements. The experiment can be described as follows (see Fig. 2): at the beginning the lifetime is about $75 \mathrm{~min}$, then a scraper is moved in until the lifetime is reduced to $40 \mathrm{~min}$. After $1 \mathrm{~min}$ the scraper is retracted by 3 $\mathrm{mm}$, which results in a period of roughly $1 \mathrm{~min}$ in which 


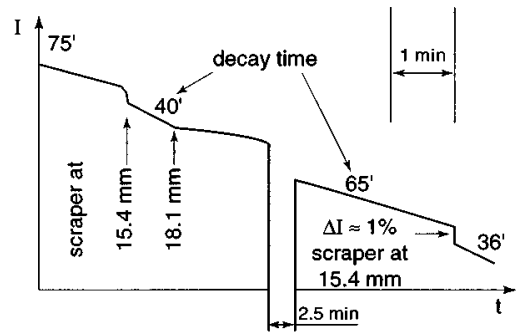

FIG. 2. Detection of slow "diffusion."

almost no losses are detected. It seems that this is the time the particles need to "diffuse" out until they reach the retracted scraper. Thereafter one finds that the lifetime stabilizes at $65 \mathrm{~min}$, which is close to the original value. Finally, the lifetime of $36 \mathrm{~min}$ is recorded (which is close to the 40 min after the first scraping) when the scraper is put back to its closest location with respect to the beam center. From this a "diffusion" rate of $3 \mathrm{~mm} / \mathrm{min}$ can be estimated. Without strong sextupoles no "diffusion" could be found.

The reduction of the long-term dynamic aperture cannot be easily explained by tracking. In fact, in the same amplitude range, the tracking predicts regular (and therefore completely stable) motion. A good candidate to describe this effect is the power supply ripple that causes a tune modulation: the combined effect of modulation and nonlinearities may reduce the dynamic aperture. In fact, in the tracking simulations [16] such an effect is found whenever a tune modulation depth of the order of some $10^{-3}$ is added. This qualitative picture of slow particle losses was later confirmed in an independent experiment in 1989 at Fermilab [17].

\section{Experimental sessions from 1989 to 1991}

In the following years we performed our experiments in view of measuring "diffusion" rates as a function of betatron amplitude, tune modulation depth (larger than the natural one), and tune modulation frequency. It turned out, however, to be much more difficult than expected to get quantitative and reproducible results from the experiment.

This is mainly due to the fact that the SPS cannot deliver a pencil beam: the particles fill a finite region of phase space, which is of the same order of magnitude as the amplitude region of interest (the amplitude range between immeasurable small "diffusion" rates and fast particle losses). Hence, to sample a certain betatron amplitude it was necessary to use a single kick instead of heating the beam with many small kicks. Moreover, it became mandatory to apply the same measurement procedure each time to arrive, at least approximately, at reproducible transverse distributions of protons. For these reasons it was very difficult to draw conclusions from lifetime measurements.

New qualitative results were obtained. The natural ripple spectrum was measured [3] to be $\Delta Q_{x}= \pm 1 \times 10^{-4}$ of which one-half can be attributed to seven ripple lines between 50 and $1000 \mathrm{~Hz}$. Furthermore, it was possible to use octupolar magnets to reduce the detuning caused by sextupoles by roughly a factor of 10 (see Fig. 3). Even more important is the induced improvement of the dynamic aperture by some $30 \%$ (this makes us confident that the detuning correction in the LHC will lead to some improvement as well). A general,

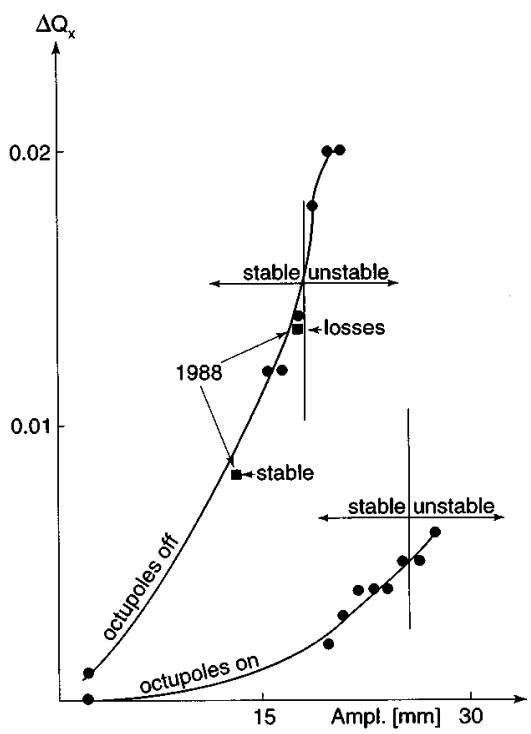

FIG. 3. Detuning and stability.

more qualitative result of the experiment was the fact that the lifetime depended strongly on the amplitude of the additional tune modulation (in agreement with the tracking simulations), but it is almost insensitive to the frequency modulation (while in simulations a smooth dependence is found). It has to be mentioned that the way the stability border was determined differs in the tracking and in the experiment: in the tracking the border of the onset of chaos was used, while in the experiment the actual particle loss was taken. A toy model (a simple FODO cell plus a sextupole) was tracked for many millions of turns (see Fig. 4) with and without tune modulation: the difference in particle losses between cases with tune modulation on or off becomes apparent after some $10^{5}$ turns, while the onset of chaotic motion shows up after only 20000 turns. The more pronounced effect of the smaller tune modulation frequencies is only visible after more than $10^{7}$ turns. This has two consequences: first it

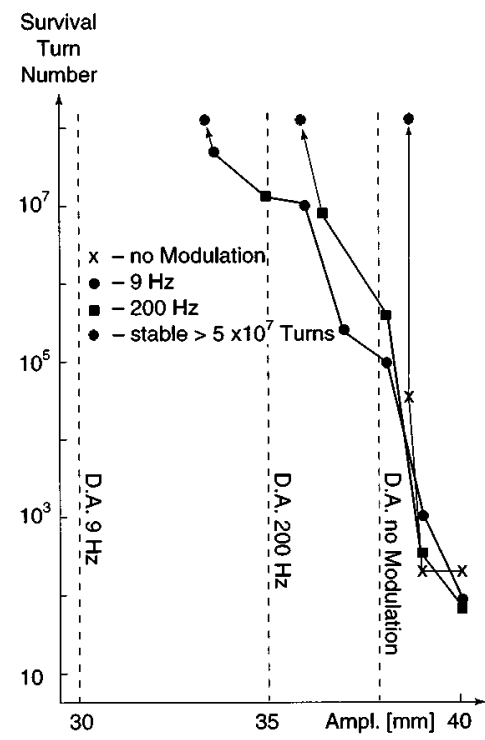

FIG. 4. Long-term stability with tune modulation. 
could very well be that the difference of the effect of the tune modulation frequencies is only visible after the storage time of interest and second, in the case with tune modulation, the onset of chaotic motion is generally much too pessimistic as a criterion for the dynamic aperture, which in our case is always the amplitude below which the motion is stable for a given time interval.

An interesting side issue was the study of the effect of two simultaneous tune modulation frequencies compared with only one frequency keeping the total tune modulation depth constant. The two tune modulation frequencies reduced the lifetime by more than a factor of 3 compared with the one-frequency case (Fig. 9 shows this experiment repeated after two years). Although these results may not be generally applicable, these findings led to a study that treated the case with more than one frequency in a more rigorous theoretical framework [18]. Another feature often found in the experiment is the appearance of the so-called "shoulder": right after a retraction of the scraper the intensity stays almost constant (infinite lifetime), and after a certain time interval, the intensity bends over rather abruptly without a smooth transition leading to a constant finite lifetime. This phenomenon was studied in Ref. [19] and found to be in contradiction with a simple diffusion mechanism. Finally we would like to mention a related study concerning slow particle losses in hadron colliders [20], which gives a phenomenological description of these losses in phase space.

\section{PREPARATION OF THE SPS AND THE TRACKING MODEL}

In this section we present the instrumentation and the experimental conditions. The tracking model for the SPS is described and the simulation methods are explained. We show that the model reproduces the basic nonlinear behavior of the SPS.

\section{A. Instrumentation for the SPS experiment}

The CERN SPS is a synchrotron with 1100-m mean radius. It can accelerate protons from $14 \mathrm{GeV}$ up to $450 \mathrm{GeV}$ for fixed-target experiments. For the dynamic aperture experiments, sizable nonlinearities were introduced by eight sextupoles (part of the slow extraction system), which are about 10 times stronger than the chromaticity sextupoles. They were grouped into two families with different polarity

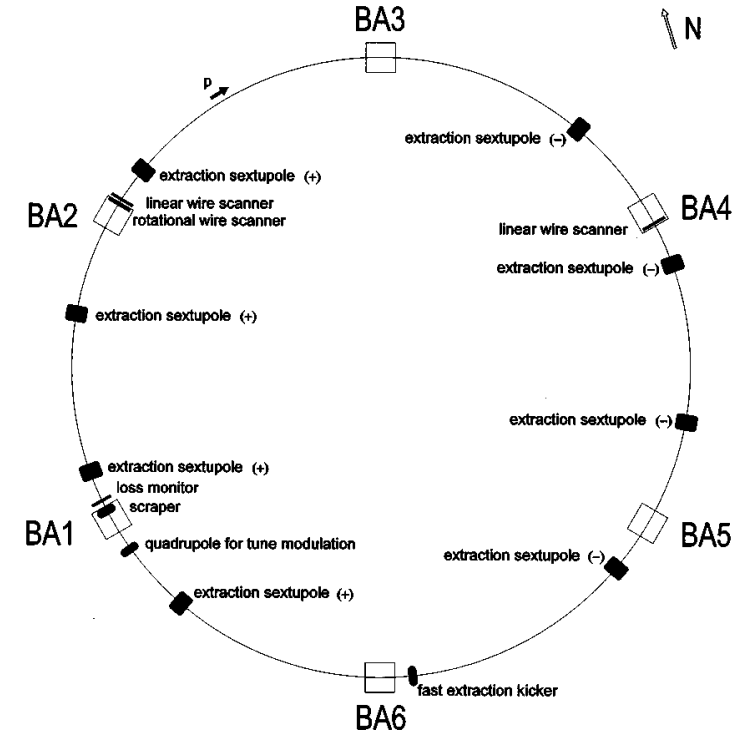

FIG. 5. Instrumentation at the SPS. The eight strong sextupoles, the quadrupole used as the source of additional tune modulation, and the kicker magnet are shown at their locations in the SPS. The positions of the linear and rotational wire scanners, the scrapers, and the loss monitor are also given. (BA stands for access hall.)

(Fig. 5) so as to avoid a change of the chromaticity and the excitation of the third-order resonances.

A single quadrupole (BA1 in Fig. 5) introduced additional tune modulation. A kicker magnet (BA6 in Fig. 5) was used to vary the average amplitude of the particle beam. Pairs of horizontal and vertical scrapers (BA1 in Fig. 5) served as aperture limiters and the losses at these scrapers were detected by a scintillator $45 \mathrm{~m}$ downstream.

Momentum and tune distributions as well as the natural ripple spectrum were measured with a Schottky system [21]. A turn-by-turn data acquisition system (see Ref. [22]) recorded position and intensity signals of a number of pickups, scraper positions and loss monitor outputs. After having applied a kick to the beam, the fast Fourier transform from the position signals gives the tunes and the line spectra due to resonances. Figure 6 shows how readings of two pickups separated by a multiple of $90^{\circ}$ allow the depiction of phasespace projections.

Two types of wire scanners were installed in the SPS (Fig. 5). In "rotational" scanners a wire, mounted on a turn-

TABLE I. Fixed beam parameters for the SPS experiment.

\begin{tabular}{ll}
\hline \hline Energy $E$ & $120 \mathrm{GeV}$ \\
$1 \sigma$ normalized emittance & $\approx 5 \mathrm{mmmrad}$ hor. and ver. \\
Intensity $I$ (beam current) & $<10^{12} \mathrm{p}(7 \mathrm{~mA})$ \\
$\mathrm{RF}$ & short-term on, long-term off \\
Momentum spread $\Delta p / p$ & $\approx 10^{-3}$ \\
Closed-orbit rms & $\leqslant 0.3 \mathrm{~mm}$ hor. and ver. \\
Linear coupling & $\left|Q_{x}-Q_{z}\right| \leqslant 0.003$ \\
Chromaticity $Q^{\prime}=\Delta Q /(\Delta p / p)$ & $\approx 1$ hor. and ver. \\
Natural tune ripple lines & $50,100,150,300,350,500,600 \mathrm{~Hz}$ \\
& sum of modulation depth $0.5 \times 10^{-4}$ \\
Total natural tune ripple depth & $1.1 \times 10^{-4}$ \\
\hline \hline
\end{tabular}




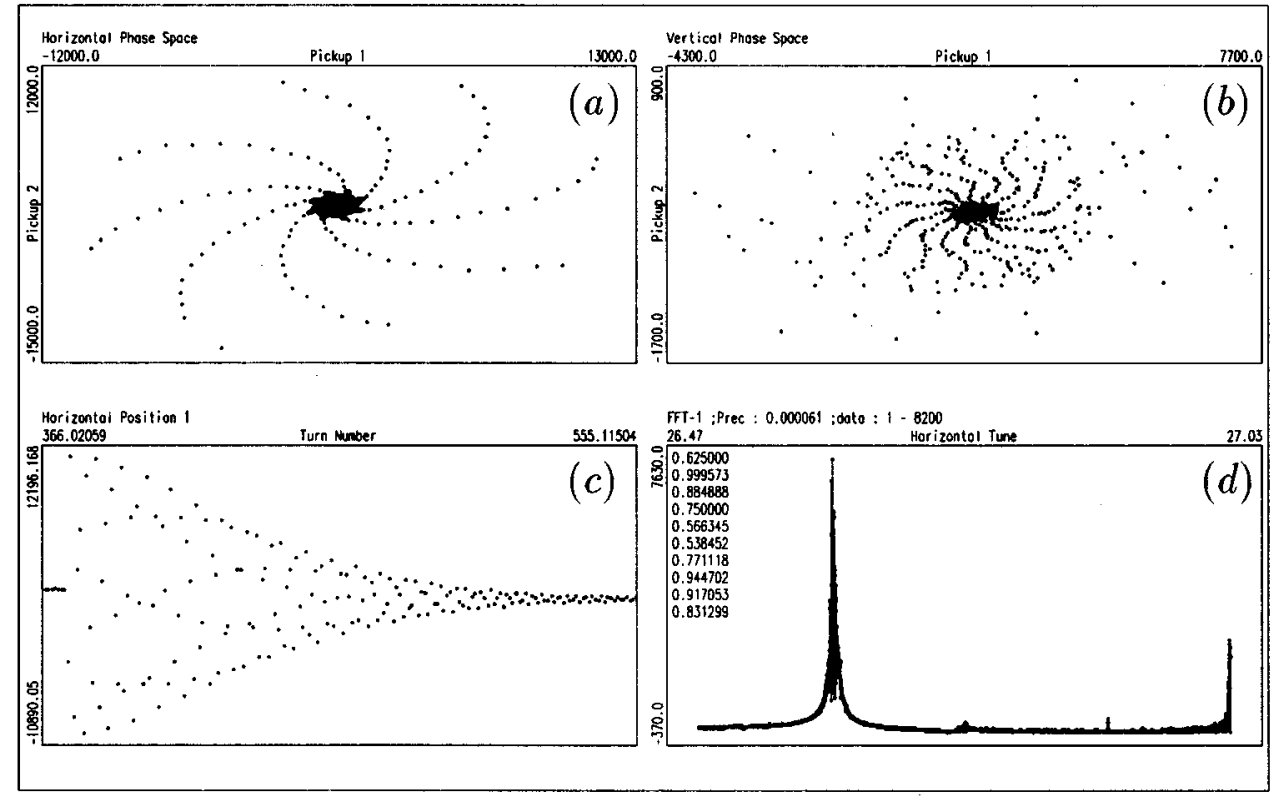

FIG. 6. Phase-space measurement. Motion is depicted close to a horizontal eighthorder resonance (a); on (b) the vertical phase-space plot is shown. The horizontally kicked and decohered beam can be seen on (c) and the horizontal FFT on (d).

able fork, went through the beam at a speed of $6 \mathrm{~m} / \mathrm{s}$ (Ref. [23]). In "linear" scanners the wire is held by a shiftable fork, passing the beam with $0.4 \mathrm{~m} / \mathrm{s}$. Secondary-particle emission caused by the beam hitting the wire can be observed using either scintillators or the depletion current in the wire itself. Since the original $36-\mu \mathrm{m}$-thick carbon wires induced an intensity drop of $2.9 \times 10^{-3}$ per scan, the wires of the "linear" scanner in BA4 were replaced by $8 \mu \mathrm{m}$ wires for both the horizontal and vertical planes. With such a thin wire the intensity loss per scan was reduced to some $0.7 \times 10^{-4}$ per scan and no beam blowup could be observed. With the position resolution of a ruler and the sensitivity of the scintillator, $5 \times 10^{7}$ protons $\left(10^{-4}\right.$ of a typical intensity) could be clearly detected at the edge of the beam profile.

\section{B. Experimental conditions}

The energy of $120 \mathrm{GeV}$ was chosen to have small remanent fields, hardly any saturation, negligible space charge effects, and at the same time a high efficiency of the nonlinear fields. The $1 \sigma$ normalized emittance, as given by the preaccelerator chain, amounts to some $5 \mathrm{~mm}$ mrad in the horizontal and vertical planes. The intensity was kept below $10^{12}$ protons to minimize the resistive-wall effect. The momentum spread was measured with the longitudinal Schottky signal to be some $10^{-3}$. In each experimental session the closed orbit, the linear coupling, and the chromaticity were corrected to very high precision.
This preparation was done in pulsed mode. Every $14.4 \mathrm{~s}$ the proton beam was injected into the SPS, accelerated to $120 \mathrm{GeV}$, and kept at this energy for $8 \mathrm{~s}$ while we carried out our studies. Once the strong sextupoles were turned on, all systems normally used to counteract collective instabilities could be switched off and the kicker was used to explore different amplitudes.

The long-term studies were performed in a continuous mode with the RF switched off (coasting beam) since RF noise could not be reduced to acceptable levels. With the continuous-tune measurement system [21] the natural ripple spectrum showed seven major lines that add up to a total modulation depth of $0.5 \times 10^{-4}$, which is half the measured total tune ripple depth. All these conditions are summarized in Table I.

In Table II the studied parameter range of the experiment is shown. The two working points WP1 and WP2 are depicted in Fig. 7 together with the detuning due to the nonlinearities. WP1 is close to a horizontal eighth-order resonance, WP2 is close to a horizontal fifth-order resonance. Both resonances are strong but do not define the dynamic aperture. However, a number of seventh-order resonances lead at both working points to particle loss.

The additional modulation frequencies $(9,40$, and 180 $\mathrm{Hz}$ ) were chosen for the following reasons: $9 \mathrm{~Hz}$ to test the low-frequency range, $40 \mathrm{~Hz}$ as a frequency close to but different from the main power supply ripple frequency of 50

TABLE II. Studied parameter range for the SPS experiment.

Working points $\left(Q_{x}, Q_{z}\right)$

Tune modulation frequencies

Horizontal tune modulation depth

Kick strength (at $\beta_{x}=100 \mathrm{~m}$ )

Horizontal scraper position
WP1 (26.637, 26.533)

WP2 $(26.605,26.538)$

others

9, 40, 180, 9+40,9+180 Hz

$0.5 \times 10^{-3}, 1.1 \times 10^{-3}, 1.87 \times 10^{-3}$

$1.9-19.5 \mathrm{~mm}$

from $10.8 \mathrm{~mm}$ to the beam pipe 


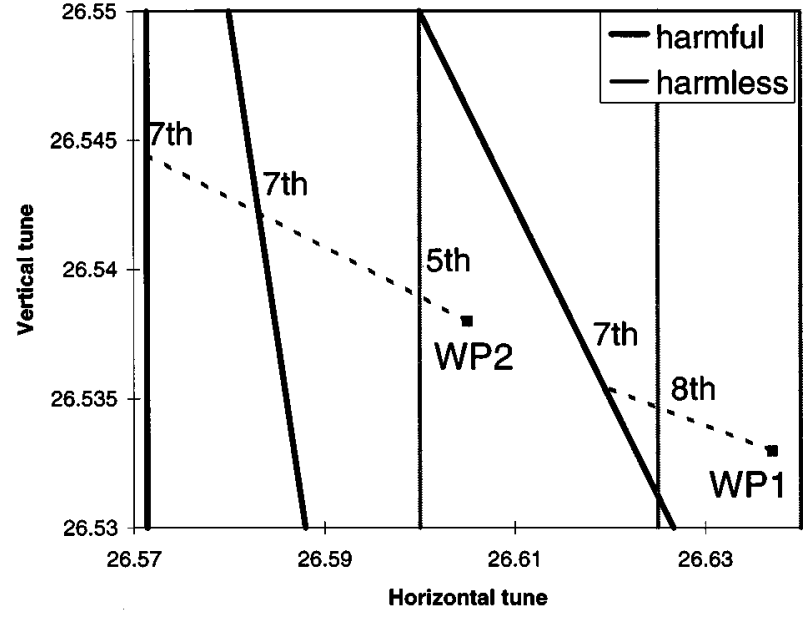

FIG. 7. The working points WP1 and WP2 in the tune diagram.

$\mathrm{Hz}$, and $180 \mathrm{~Hz}$ as a typical synchrotron frequency. Experiments were also made with combinations of two frequencies. The horizontal tune modulation depth was $0.5 \times 10^{-3}$, $1.1 \times 10^{-3}$, and $1.87 \times 10^{-3}$, the larger values being applied at WP1 to obtain loss rates similar to those at WP2. The ratio of horizontal and vertical tune modulation depth was $\Delta Q_{x} / \Delta Q_{z}=1.75$ given by the beta functions at the modulating quadrupole.

The kick strength was varied to probe different amplitudes up to the dynamic aperture. Furthermore, the scrapers were used to precisely define the edge of the beam.

\section{Tracking model and methods}

The computer code SIXTRACK [24] was used for the tracking studies. Drifts, dipoles, and quadrupoles were modeled as thick lenses; all other elements (skew quadrupoles and sextupoles) were approximated as thin lenses. Apart from the eight strong sextupoles, the 108 chromaticity correction sextupoles were included in the model. The effect of the closed orbit was introduced by shifting the sextupoles by the measured values, the linear coupling was adjusted to the measured one using one family of six skew quadrupoles, and the chromaticity was corrected to zero. Particles were distributed in amplitude and momentum to probe a sufficient fraction of the phase space. The scraper was used as observation point to detect the losses.

One of the tools to analyze and understand phenomenologically the intricate nature of particle motion in phase space is an elaborate postprocessing of the tracking data [25]. The methods applied and the quantities computed are listed below. Tune: The tune was determined with a fast Fourier transform and by averaging the phase advance per turn. Local chaos: The time evolution of the distance of initially close-by particles allows one to distinguish between regular and chaotic motion. Chaotic motion manifests itself in an exponential growth of this distance, which grows linearly for regular motion. Survival plots: The loss time versus amplitude (denoted as survival plot, see Ref. [26]) was our main tool for determining the dynamic aperture in the simulations. Typically the loss time increases exponentially with decreasing amplitude, and very close to the regular region it tends to infinity. Averaging: When tune modulation was switched on, the phase advance was averaged over one tune-modulation period to derive meaningful numbers for the tunes. The particle amplitudes were averaged as well over that period to study their mean and rms values as a function of time.

All simulation studies were performed on an IBM RISC station cluster. For one turn in the SPS $(0.023 \mathrm{~ms}$ storage time) a computing time of about $1.5 \mathrm{~ms}$ per particle pair was needed on this system. This means that for a typical longterm run with 32 particle pairs and the maximum turn number of $15 \times 10^{6}$ turns (equivalent to 6 -min storage time) a CPU time of roughly two weeks was needed.

\section{Amplitude dependence of betatron tunes}

Since the amplitude-dependent tune is an important property of any nonlinear oscillator system, a disagreement between tracking and experiment would cast doubt on the validity of the tracking model.

Particle motion in hadron accelerators can be described in the Hamiltonian formalism [27]. Transforming the usual accelerator coordinates into action-angle variables so that the phase-dependent part of the Hamiltonian can be neglected, the lowest-order part of the nonlinear Hamiltonian can be written as

$$
H=a I_{x}^{2}+2 b I_{x} I_{z}+c I_{z}^{2},
$$

where $I_{x}$ and $I_{z}$ are the horizontal and vertical action, respectively, and $a, b$, and $c$ the so-called detuning coefficients. The detuning can therefore be expressed as

$$
\begin{aligned}
& \Delta Q_{x}=2 a I_{x}+2 b I_{z}, \\
& \Delta Q_{z}=2 b I_{x}+2 c I_{z} .
\end{aligned}
$$

In our case, where sextupoles are the dominant nonlinear elements, the coefficients $a, b$, and $c$ in Eqs. (2) and (3) can be derived from the beta functions and sextupole strength in second-order perturbation theory [27].

In Fig. 8 the detuning as a function of action is depicted for the model and the experimental data at WP1, respectively. Data at the working point WP2 are similar. As expected from Eqs. (2) and (3) the increase of the detuning is linear with action. The extensive experimental data agree very well with the expectation from the model. Table III

TABLE III. Detuning coefficients at WP1 and WP2.

\begin{tabular}{cccc}
\hline \hline & $a$ & $b$ & $c$ \\
& {$\left[10^{-3}\left(\mathrm{~mm} \mathrm{mrad}^{-1}\right]\right.$} & {$\left[10^{-3}(\mathrm{~mm} \mathrm{mrad})^{-1}\right]$} & {$\left[10^{-3}(\mathrm{~mm} \mathrm{mrad})^{-1}\right]$} \\
\hline WP1 & -5.2 & 1.1 & -0.3 \\
WP2 & -5.6 & 1.2 & -0.3 \\
\hline \hline
\end{tabular}


summarizes all detuning coefficients, where $a$ and $b$ are taken from the experiment and the model, while the term $c$ was not measured.

\section{EXPERIMENTS BASED ON INTENSITY MEASUREMENTS}

The detection of slow particle losses in the experiments of 1989 was the starting point for an extensive series of experiments to study particle losses as a function of betatron amplitude, modulation depth, and frequency. Emphasis was put on obtaining quantitative results.

Results from these early experiments, in which the beams were heated with many small kicks, suggested that the loss process could be described by a diffusion model. Considering an amplitude- or action-dependent diffusion coefficient $D\left(I_{x}\right)$ the appropriate diffusion equation reads

$$
\frac{\partial f\left(I_{x}, t\right)}{\partial t}=\frac{\partial}{\partial I_{x}}\left(D\left(I_{x}\right) \frac{\partial f\left(I_{x}, t\right)}{\partial I_{x}}\right) .
$$

Besides loss measurements at the two working points in which the modulation frequency and amplitude were systematically varied the influence of two simultaneously applied modulation frequencies was examined. A total of 61 experimental runs were recorded (a complete list can be found in Appendix B of Ref. [28]).

\section{A. Measurements}

For reasons of reproducibility experiments were always performed in the following way: first, a single kick of welldefined strength was applied thus placing the maximum of the particle distribution at the desired amplitude. The resulting distribution could be estimated analytically (see Appendix A in Ref. [28]). Second, a scraper was used to define a maximum betatron amplitude and, after a subsequent retraction, it acted as the physical aperture. Third, for reasons of reproducibility, a complex experimental procedure had to be strictly followed (cf. Fig. 9): the horizontally kicked beam was scraped vertically to remove the vertical beam tail (time $T_{-3}$, intensity $\left.I_{-3}\right)$. Then the vertical scraper was retracted, the horizontal scraper was moved in $\left(T_{-2}, I_{-2}\right)$, and the additional tune modulation was switched on $\left(T_{-1}, I_{-1}\right)$. After waiting until a stationary particle distribution with an approximately constant loss rate was produced, the horizontal scraper was retracted $\left(T_{0}, I_{0}\right)$, typically by $1 \mathrm{~mm}$, to create an amplitude region free of particles that was expected to be filled by some transport process. The losses were assumed to stabilize after a time that is denoted by $T_{1 b}$ leading to a new stationary particle distribution. $T_{1 a}$ was determined from the point of intersection of the linear slope of the intensity with the line $I_{0}=$ const. After some $15 \mathrm{~min}$ the tune modulation was switched off and the beam was scraped once more vertically $\left(T_{2}, I_{2}\right)$ and horizontally $\left(T_{3}, I_{3}\right)$ to determine the plane in which the particle losses predominantly took place.

In the experimental period of 1993 that concluded this type of experiment, the following parameters were varied: At WP1 and WP2 three frequencies $(9,40$, and $180 \mathrm{~Hz})$ and the tune modulation depths $1.1 \times 10^{-3}$ and $1.87 \times 10^{-3}$ were tested, in addition at WP1 the tune modulation depth

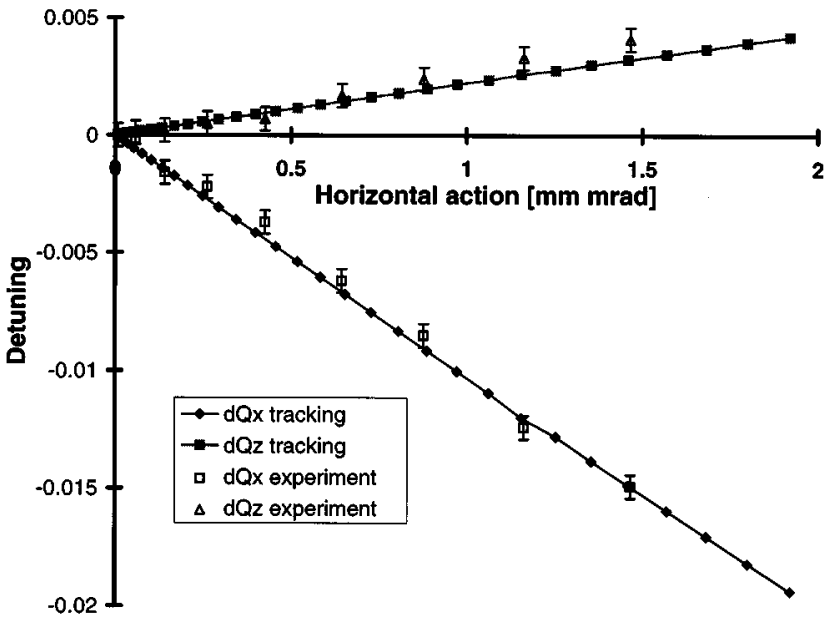

FIG. 8. Detuning as a function of action at WP1.

$\Delta Q_{x}=0.55 \times 10^{-3}$ was studied.

We will discuss results from WP2 since in this case all tune modulation depths and frequencies were tested. In Table IV the loss $L$ taken $15 \mathrm{~s}$ after switching on the tune modulation and the time interval $T_{1 a}-T_{0}$ is shown, which corresponds to the average transport time between the beam edge and the retracted scraper position. This time interval was the quantity originally measured in the experiment of 1989. Table IV also holds an error if the number of cases $n$ is larger than 1.

A larger modulation depth leads to a larger loss $L$ but the errors are so large that one cannot quantify reliably the difference between the different modulation depths and frequencies. The time $T_{1 a}-T_{0}$ obtained for the smaller modulation depth has similar errors and for the larger depth it was even impossible to determine this value.

These difficulties to obtain quantitative results persisted in spite of the efforts to specially prepare the SPS, to calibrate all instruments, and to apply an elaborate measurement procedure, the limiting parameter being the large transverse beam size. We therefore concluded that any advances in this line of experiment would require a much smaller particle distribution in amplitude.

\section{B. Discussion of diffusion models}

Assuming a diffusion model a diffusion coefficient in Eq. (4) must be found that would allow one to describe the experimental intensity curves. According to the experimental findings of earlier years any model description of these curves has to include a strong amplitude dependence of the diffusion coefficient (such as, for instance, $I_{x}^{m}$ ) and an amplitude $\left(I_{x 0}\right)$ below which only a background mechanism leads to particle loss. For example, rest gas scattering leads to a diffusion coefficient $D\left(I_{x}\right)=D_{0} I_{x}$ [29]. One therefore arrives at a four-parameter model similar to that used in Ref. [8]:

$$
D\left(I_{x}\right)=\left\{\begin{array}{l}
D_{0} I_{x} \quad \text { if } I_{x}<I_{x 0}, \\
D_{0} I_{x}+D_{1}\left(I_{x}-I_{x 0}\right)^{m} \quad \text { if } I_{x 0}<I_{x} .
\end{array}\right.
$$




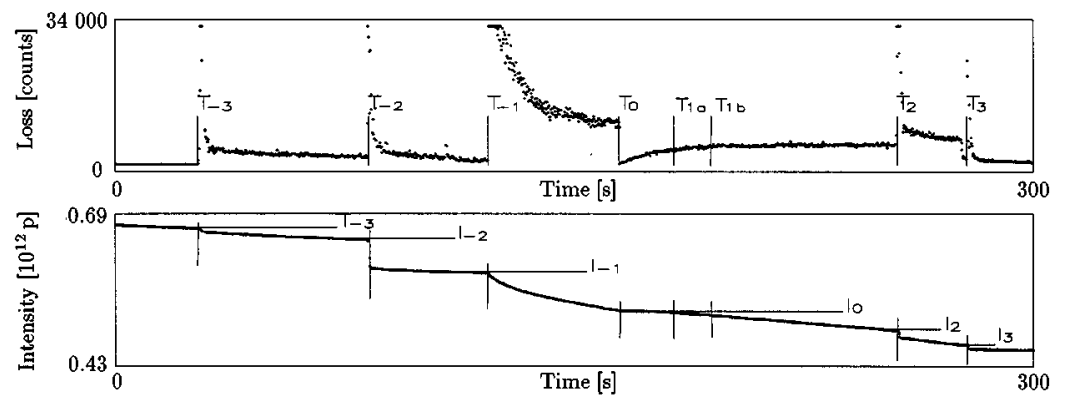

FIG. 9. Loss rate and beam intensity as a function of time in the scraper experiment. ( $p$ denotes protons.)

First a simple approach was tested, namely, fitting a diffusion coefficient that increases linearly with action to the experimental data. Unfortunately, this fitting procedure did not converge in all analyzed cases. Due to the discouraging results obtained one had to refrain from a more detailed analysis using the four parameters in Eq. (5). Moreover, detailed tracking studies (Sec. VI) seem to indicate that a diffusion model like Eq. (5), which is valid over the whole phase, does not fit the phenomenology of the loss processes.

In contradiction to measurements at the HERA proton ring [29], where a diffusion model was successfully employed for the description of particle losses during luminosity runs, the irreproducibility of the experimental results in the SPS experiment prevents a quantitative determination of a diffusion coefficient and a decision if a diffusion model is appropriate to describe the loss mechanism. However, the main source for nonlinearities in the case of HERA was the beam-beam interaction whereas sextupoles are the dominating nonlinear elements in the SPS.

\section{Effect of two modulation frequencies}

In 1991 it was observed that the lifetime dropped to about one-third when two frequencies $(9$ and $180 \mathrm{~Hz}$ ) were applied instead of only one $(9 \mathrm{~Hz})$ when the same total tune modulation depth was kept. The same procedure was tested in 1993 at another working point [see Fig. 10(a), WP1] and for comparison the experiment at the original working point was repeated [Fig. 10(b)], confirming the previous result. The interesting observation is that the effect of two frequencies in case (a) is less pronounced than in case (b).

The effect of two modulation frequencies can be understood qualitatively as follows [18]. With only one modula-

TABLE IV. Experimental particle stability at WP2. The particle loss after $15 \mathrm{~s}$ following the switch-on time of the tune modulation is denoted by $L$. Time $T_{1 a}$ is determined from the point of intersection of the linear slope of the intensity with the line $I_{0}=$ const, $n$ is the number of experimental runs with the same modulation frequency and depth. The given error is the $n-1$ standard deviation of the mean value. All experimental cases were done with the same kick strength and scraper positions.

\begin{tabular}{lccccc}
\hline \hline & \multicolumn{3}{c}{$\Delta Q_{x}=0.55 \times 10^{-3}$} & \multicolumn{3}{c}{$\Delta Q_{x}=1.1 \times 10^{-3}$} \\
& $L(\%)$ & $T_{1 a}-T_{0}(\mathrm{~s})$ & $n$ & $L(\%)$ & $n$ \\
\hline $9 \mathrm{~Hz}$ & $5.2 \pm 1.1$ & $13.4 \pm 5.6$ & 3 & $5.8 \pm 3.3$ & 7 \\
$40 \mathrm{~Hz}$ & 6.4 & 13.5 & 1 & $8.9 \pm 4.5$ & 3 \\
$180 \mathrm{~Hz}$ & $6.7 \pm 2.4$ & $17.0 \pm 7.3$ & 6 & $12.3 \pm 6.9$ & 4 \\
\hline \hline
\end{tabular}

tion frequency the resonance condition reads

$$
k Q_{x}+l Q_{z}+m \frac{f_{\text {mod }}}{f_{\text {rev }}}=p,
$$

$f_{\text {mod }}$ being the modulation frequency and $f_{\text {rev }}$ the revolution frequency, i.e., resonance sidebands are created around the main resonance $k Q_{x}+l Q_{z}=p$. For high modulation frequencies the sidebands have a large spacing; for low frequencies the spacing is small and the sidebands may overlap so that particles can be transported over wide amplitude regions. With two modulation frequencies the resonance condition is

$$
k Q_{x}+l Q_{z}+m \frac{f_{\mathrm{mod} 1}}{f_{\mathrm{rev}}}+n \frac{f_{\mathrm{mod} 2}}{f_{\mathrm{rev}}}=p .
$$

Having a high and a low modulation frequency (in our case $f_{\text {mod1 }}=180 \mathrm{~Hz}$ and $f_{\bmod 2}=9 \mathrm{~Hz}$ ) the high-frequency $f_{\bmod 1}$ leads to largely spaced sidebands $k Q_{x}+l Q_{z}$ $+m\left(f_{\bmod 1} / f_{\text {rev }}\right)=p$ around the main resonance. Around those principal sidebands additional sidebands due to the low-frequency $f_{\text {mod2 }}$ are created that can overlap.
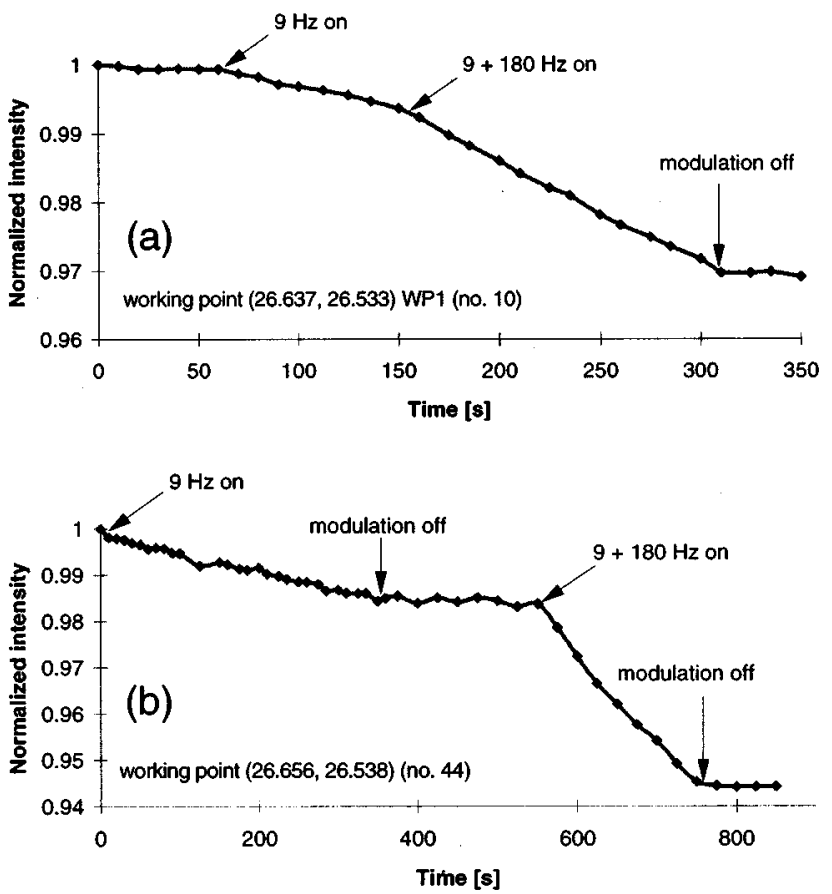

FIG. 10. Comparison between one and two modulation frequencies. 


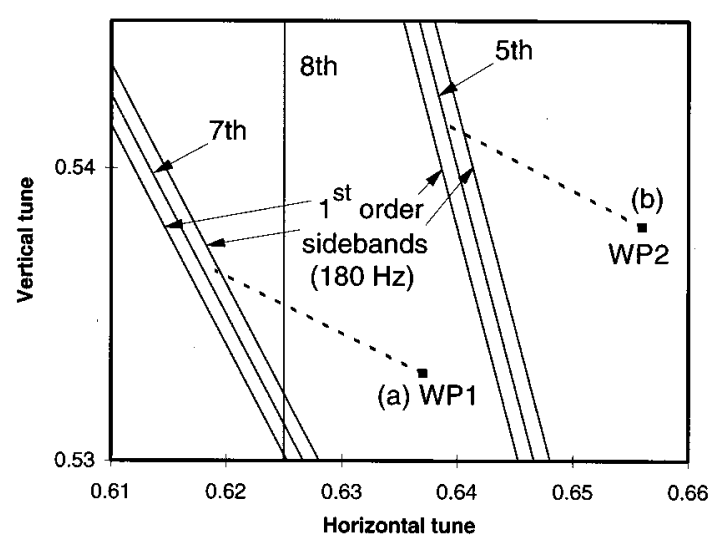

FIG. 11. Tune diagram for the cases where two modulation frequencies were applied simultaneously.

In Fig. 11 the working points for both cases are shown together with the detuning lines (dotted) up to the dynamic aperture. In case (a) at WP1 the dynamic aperture is close to a seventh-order resonance, in case (b) it is close to a fifthorder resonance. In both cases only the first-order resonance sidebands due to the higher frequency $(180 \mathrm{~Hz})$ are depicted, which are by far the strongest sidebands for the chosen modulation depth and frequency. One of the sidebands at each working point can reach the particle distribution.

From tracking data the resonance driving term of the fifth-order resonance in case (b) was found to be about a factor 500 larger than the driving term of the seventh-order resonance in case (a). We therefore expect that the island sizes (which are of the order of the square root of the resonance driving terms) of the resonance sidebands around the first-order $180-\mathrm{Hz}$ sidebands in case (b) are much larger than those in case (a). As a consequence, more of these sidebands can overlap and the amplitude region of overlapped sidebands is larger leading to a stronger particle loss in case (b).

\section{DYNAMIC APERTURE EXPERIMENTS}

In the field of nonlinear accelerator dynamics various definitions of the dynamic aperture are in use. A relevant definition for machine operation is the amplitude below which no sizable particle losses take place for a time interval of interest. The dynamic aperture is a particular concern for the LHC at injection time when nonlinearities and beam size are at their maximum. Particles have to survive under these unfavorable conditions for about $15 \mathrm{~min}$. The main practical aim of the SPS experiment was to test if tracking simulations can predict the dynamic aperture of a machine in conditions similar to those of the LHC, as far as nonlinearities are concerned.

In our studies we had to face two principal limitations: first, the full time interval of interest (15 min storage time or $40 \times 10^{6}$ turns in the SPS) could not be fully explored with tracking simulations, only one-third (345 s) could be reached with reasonable computing effort. Second and more relevantly, the SPS experiment was rather restricted by the large natural beam size. This precludes any fine exploration of the phase space as is done in tracking by varying the amplitude in small steps. In particular, small chaotic regions within an otherwise stable region cannot be detected. How-

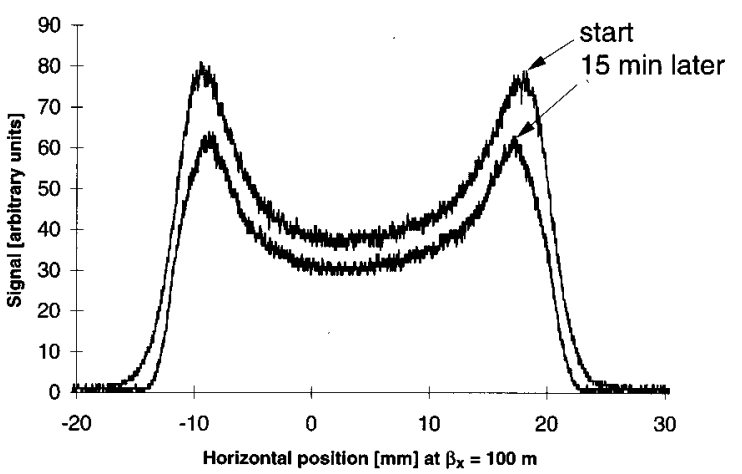

FIG. 12. Time evolution of a wire scan profile.

ever, the maximum amplitude above which no particles survive after a certain time gives a good measurement of the stability limit.

\section{A. Measurements}

The almost nondestructive scanner system with $8-\mu \mathrm{m}$ flying wires was used for the dynamic aperture measurements. The wire scanner profiles are the projections of the particle distribution in phase space onto the $x$ and $z$ axes, respectively, and the time-dependent bottom width of those profiles gives information to be compared with survival plots obtained from simulations (Fig. 12). Scrapers defined the beam edge and, once taken out, they served as a known physical aperture limiter.

Taking advantage of the experience from the scraper experiment, which showed that the variation of the modulation frequency had hardly any effect on the loss rates, we restricted ourselves to a single modulation frequency of $9 \mathrm{~Hz}$. We used modulation depth that gave comparable and welldetectable particle losses at both working points (i.e., $\Delta Q_{x}=1.87 \times 10^{-3}$ at WP1 and $\Delta Q_{x}=0.55 \times 10^{-3}$ at WP2, respectively).

In Fig. 13(a) four wire-scan measurements at WP1 with additional tune modulation are displayed. These curves have been smoothed and the estimated error bars are shown in case No. 91. The reproducibility of the experimental results has been found to be within $2 \%$ for two measurements performed under the same conditions but five months apart (No. 62 and No. 76). Owing to the rather large beam size the results depend on the measurement procedure: cases No. 62 and No. 76 were scraped horizontally after kicking, whereas cases No. 78 and No. 91 were left unscraped and in addition the latter case was kept for some extra 15 min without additional tune modulation. Although these cases strongly differ initially, after $345 \mathrm{~s}$ the differences reduce to about 5\%.

Figure 14(a) shows the situation for WP2 where the applied ripple depth was about four times smaller. Two cases (No. 72 and No. 74) are not scraped, case No. 82, which was scraped horizontally, shows the interesting feature of the beam size growing at first and then shrinking again. Also at WP2 the differences between the various cases after $345 \mathrm{~s}$ are largely reduced (less than $3 \%$ ).

Finally Figs. 13(b) and 14(b) show the experimental results with natural ripple only. The results of all measured cases are summarized in Table V. 
(a) tune modulation of $9 \mathrm{~Hz}$ and $\Delta Q_{x}=1.87 \times 10^{-3}$

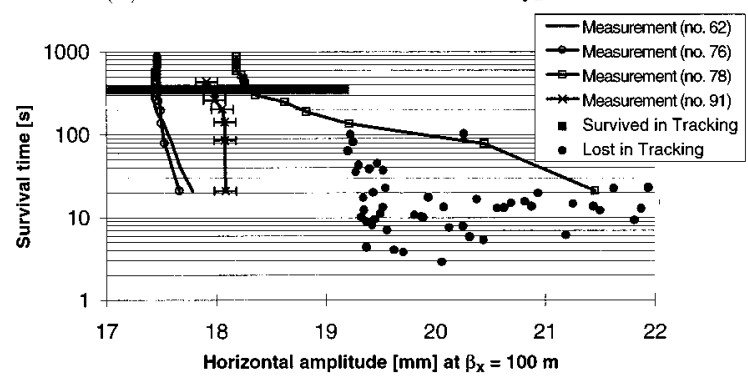

(b) natural ripple only

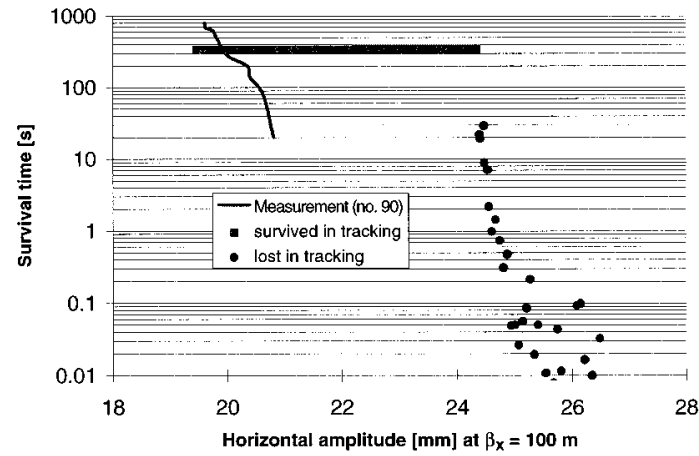

FIG. 13. SPS dynamic aperture studies at WP1. Measurement and computational results for the dynamic aperture with and without additional tune ripple at WP1 are shown in (a) and (b), respectively.

\section{B. Simulations}

As described in Sec. III C, survival plots are used to compute the dynamic aperture. According to the experimental conditions, the particles are tracked with large horizontal displacements while a vertical displacement of roughly $1 \sigma$ of the vertical beam size was considered.

In this experiment, where relatively large tune modulations are present, the onset of chaos is a much too pessimistic indicator for long-term stability. In all tracked cases a wide amplitude range could be found (column three in Table V) where chaotic (after 200000 turns) and regular regions alternate. For each chaotic region in that amplitude range a particular sum resonance is found, which apparently causes the unstable behavior. At WP1 [Fig. 13(a)] the lost particles correspond to a coupled seventh-order resonance or [Fig. 13(b)] to a horizontal 13th-order resonance. At WP2 [Figs. 14(a) and $14(\mathrm{~b})]$ the losses at large amplitudes correspond to a (a) tune modulation of $9 \mathrm{~Hz}$ and $\Delta Q_{x}=0.55 \times 10^{-3}$

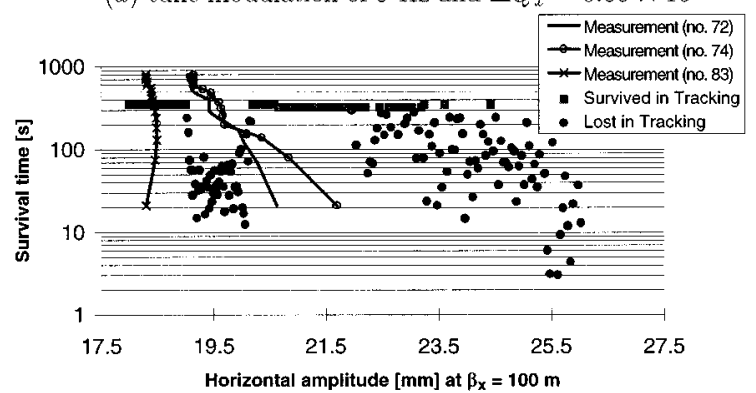

(b) natural ripple only

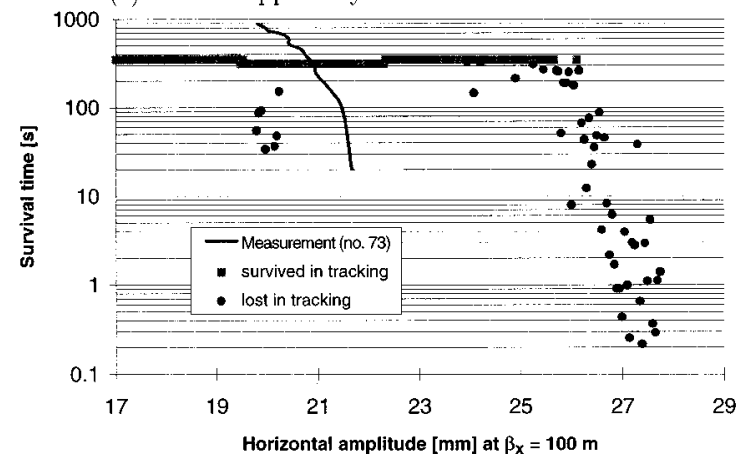

FIG. 14. SPS dynamic aperture studies at WP2. Measurement and computational results for the dynamic aperture with and without additional tune ripple at WP1 are shown in (a) and (b), respectively.

horizontal seventh-order resonance and the losses at about 20 $\mathrm{mm}$ are due to another coupled seventh-order resonance. These resonances can be found in Fig. 7.

The dynamic aperture, computed as explained, is shown in column four in Table $\mathrm{V}$ and the last column in this table shows the relative difference in percent between the computed and the measured dynamic aperture. In the cases with additional tune modulation, the difference is about $10 \%$, but without ripple this number rises to more than $20 \%$. Moreover, in the tracking we find a broad region of apparently regular motion outside the experimental stability border (not present in the cases with additional tune modulation). From this we have to conclude that destabilizing effects are missing in our tracking model. A very rough estimate for the scale of those effects is given by the strength of the additional tune modulation because the experimental dynamic aperture without extra tune modulation agrees well with that

TABLE V. Comparison of measured and computed dynamic aperture. All values are given for $\beta_{x}=100$ $\mathrm{m}$. For the onset of chaos there are two values: the larger one is the border above which no regular particles could be found; the lower (in brackets) is the lowest amplitude at which large-scale chaotic motion sets in.

\begin{tabular}{lcccc}
\hline \hline Case & $\begin{array}{c}\text { Measured } \\
\text { dynamic } \\
\text { aperture } \\
(\mathrm{mm})\end{array}$ & $\begin{array}{c}\text { Onset of chaos } \\
\left(2 \times 10^{5} \text { turns }\right)\end{array}$ & $\begin{array}{c}\text { Loss border } \\
\left(1.5 \times 10^{7} \text { turns }\right)\end{array}$ & $\begin{array}{c}\text { Comparison between } \\
\text { measurement and } \\
\text { loss border } \\
(\%)\end{array}$ \\
\hline WP1, $9 \mathrm{~Hz}, \Delta Q_{x}=1.87 \times 10^{-3}$ & 17.4 & $(7.7) 14.3$ & $1 \mathrm{~mm})$ & 10 \\
WP2, 9 Hz, $\Delta Q_{x}=0.55 \times 10^{-3}$ & 19.5 & $(7.4) 8.3$ & 22.3 & 14 \\
WP1, natural ripple only & 20.0 & $(15.7) 23.1$ & 24.4 & 22 \\
WP2, natural ripple only & 20.9 & $(9.8) 23.6$ & 25.7 & 23 \\
\hline \hline
\end{tabular}




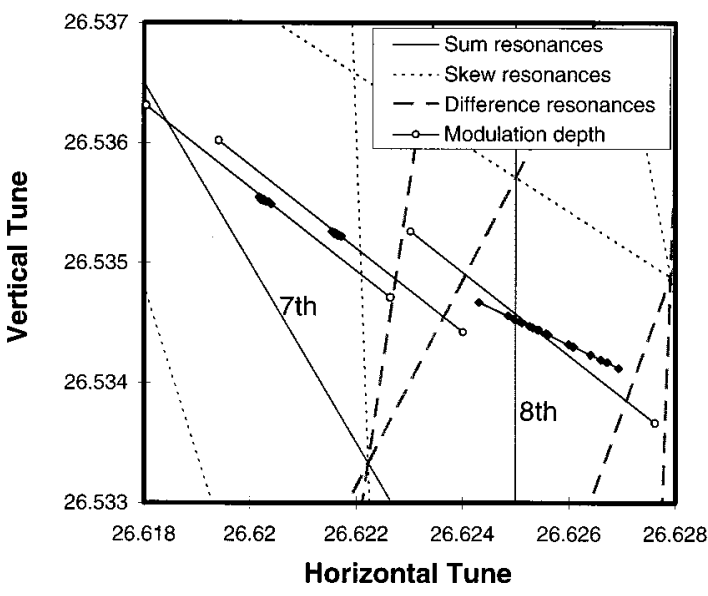

FIG. 15. Tune diagram and detuning with amplitude WP1.

of the tracking when this ripple is introduced (cf. the last two entries in column two with the first two entries in column four of Table V). The nature of these effects is still unknown. However, both neglected systematic nonlinearities and extra tune modulation are unlikely candidates. The former would lead to measurable detuning with amplitude, while for the latter the tune modulation can be understood from the measured voltage ripple of the SPS power supplies [30] and in addition it has been measured with good precision with a phase-locked loop. Some more discussion of possible causes can be found in Ref. [28].

In the complementary experiment at the HERA proton ring mentioned in Sec. II A the difference between measured and computed dynamic aperture amounts to values similar to those in the SPS [7] given that the experimental conditions were well understood.

\section{PHENOMENOLOGY OF CHAOTIC PARTICLE MOTION}

One of the main purposes of our studies was to further the phenomenological understanding of the intricate nature of the particle motion in phase space, thus continuing earlier studies [20]. In particular we were interested in the chaotic regime generated by the interplay of nonlinearities and harmonic tune modulation.

Simulation studies at WP1, based on the methods described in Sec. III C, are presented first. The subsequent section describes experimental results that successfully demonstrate the phenomena first found in the tracking.

\section{A. Simulations}

For working point WP1 and a tune modulation of $9 \mathrm{~Hz}$ and $\Delta Q_{x}=1.87 \times 10^{-3}$ we studied three betatron amplitudes $\left(16.8,18.8\right.$, and $19.5 \mathrm{~mm}$ at $\left.\beta_{x}=100 \mathrm{~m}\right)$ close to the longterm dynamic aperture. All sum, skew, and difference resonances in the amplitude regime of interest are shown up to order 13 in Fig. 15.

In the tracking studies the particle stability was tested with three methods. First, we computed the angular distance of a pair of initially close-by particles after 20000 turns (Table VI) and we found the most chaotic behavior at the smallest amplitude. Second, we followed the tunes averaged over one modulation period (5000 turns) for one particle. Twenty tune values so obtained at each of the three amplitudes are depicted in Fig. 15 together with the tune modulation depth. At the smallest amplitude (rightmost in Fig. 15) the eighth-order resonance is crossed due to the tune modulation leading to large fluctuations of the averaged tunes. The presence of this resonance also explains the strong chaotic behavior found with the first method. The largest amplitude (leftmost in Fig. 15) just reaches the seventh-order resonance beyond which strong particle loss takes place. Note that the tunes follow closely the detuning curve shown in Fig. 8. Third, we started 640 particles with initial conditions distributed over a very small phase space region at each of the three amplitudes and tracked them for $3 \times 10^{6}$ turns. It is necessary to consider such distributions rather than single particles on account of the chaotic nature of the motion in the studied amplitude range in phase space.

The amplitude evolution for the three starting amplitudes is shown in Fig. 16 using 32 of the 640 particles. The amplitudes are averaged over one tune-modulation period. At the lowest amplitude [Fig. 16(c)] the particles quickly fill (less than $10^{5}$ turns) an amplitude band around the eighthorder resonance and stay within the band. The width of this band could be identified as the amplitude region of overlapping sidebands due to the tune modulation with $9 \mathrm{~Hz}$ and $\Delta Q_{x}=1.87 \times 10^{-3}$ [28]. Not one particle out of 640 was lost (Table VI). For the second amplitude [18.8 mm, Fig. 16(b)] the amplitudes spread slowly $\left(10^{5}-10^{6}\right.$ turns). Eventually they either reach the seventh-order resonance, after which they are extracted in some $10^{4}$ turns, or they are attracted down to the eighth-order resonance: in Fig. 16(b) several particles show a decrease in their amplitude after 1.9 million turns to occupy an amplitude range which corresponds to that of part (c). The particles starting at $19.5 \mathrm{~mm}$ [Fig. 16(a)] fill the same band as the particles starting at $18.8 \mathrm{~mm}$. But since they start very close to the upper-band border, most

TABLE VI. Particle stability at three different starting amplitudes.

\begin{tabular}{lccc}
\hline \hline $\begin{array}{l}\text { Horizontal amplitude }(\mathrm{mm}) \\
\text { at } \beta_{x}=100 \mathrm{~m}\end{array}$ & 16.8 & 18.8 & 19.5 \\
$\begin{array}{l}\text { Separation angle }(\pi) \text { of 2 particles } \\
\text { after } 20000 \text { turns }\end{array}$ & 0.9 & $0.45 \times 10^{-4}$ & $0.8 \times 10^{-3}$ \\
$\begin{array}{l}\text { Lost particles } \\
\text { out of } 640\end{array}$ & 0 & 102 & 502 \\
$\begin{array}{l}\text { Amplitude rms value }(\mathrm{mm}) \\
\text { after } 1.5 \times 10^{6} \text { turns }\end{array}$ & 0.82 & 0.29 & 0.28 \\
\hline \hline
\end{tabular}


(a)
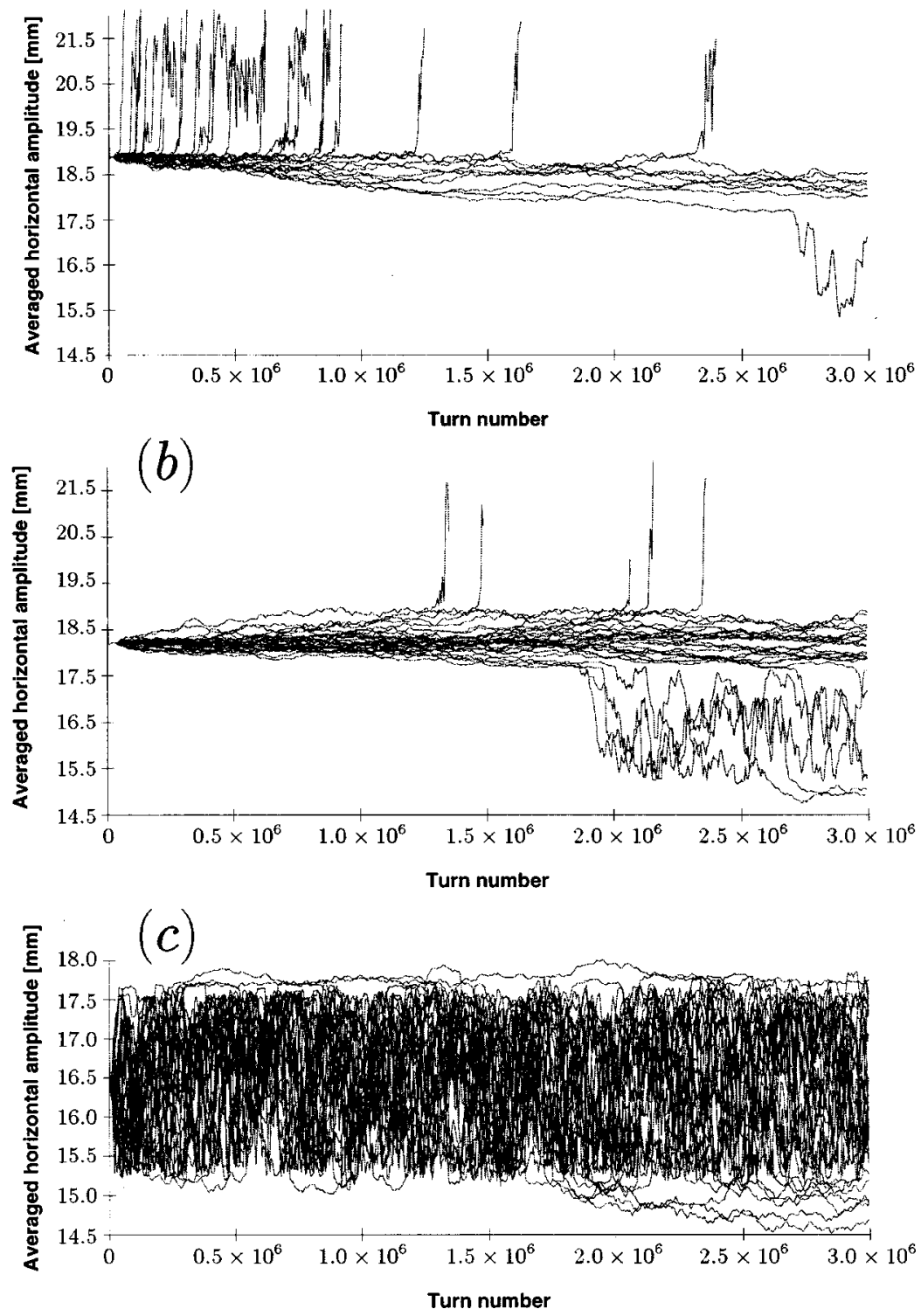

FIG. 16. Amplitude evolution of particle distributions. particles are lost and only a few are attracted to the eighthorder resonance.

Figure 17 shows the evolution of the amplitude rms values for the three cases. The very chaotic particles starting at $16.8 \mathrm{~mm}$ show an immediate increase of the rms value,

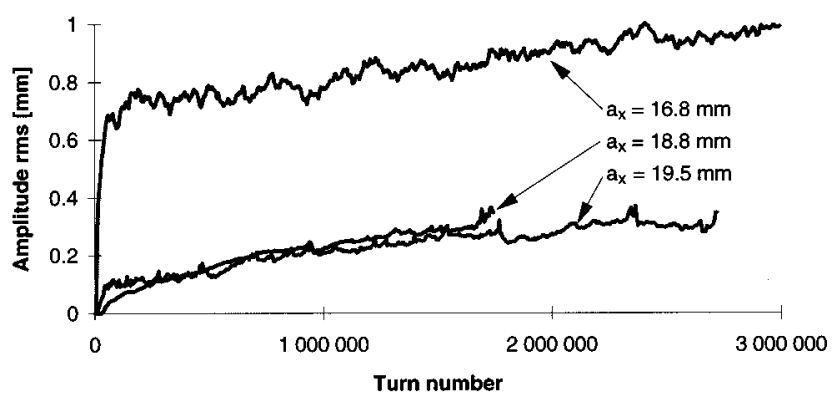

FIG. 17. Time-dependent amplitude rms values. (The same amplitudes are chosen as in Fig. 16.) which is not present in the other cases. For these amplitudes the rms values are only given up to $1.7 \times 10^{6}$ and $2.7 \times 10^{6}$ turns, respectively, since thereafter a sizable loss or attraction to the eighth-order resonance sets in, which perturbs the evolution of the rms value.

Our observations of the chaotic particle motion in the phase space at WP1 can be summarized as follows: at small amplitudes there is a band of strongly chaotic particles, which nevertheless survive for very long periods. This band is separated from a region of larger amplitudes where individual particles either slowly grow or decrease their betatron amplitude until they reach the bounds of the band. Finally, beyond a certain amplitude, a rapid particle loss takes place. It must be mentioned that a band structure was also found at WP2. This scenario is in clear contradiction with a global diffusion model. However, the amplitude evolution in time may be appropriately described by using, first, a separate (amplitude-dependent) diffusion coefficient for each band, and, second, transition probabilities between these bands. 


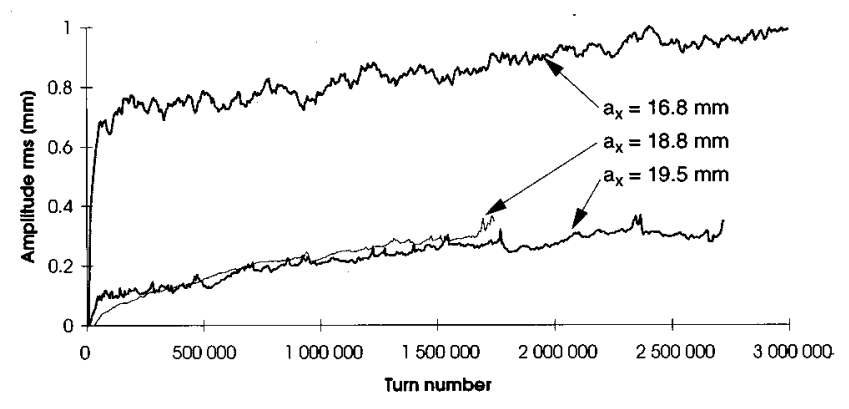

FIG. 18. Measurements of horizontal beam profile width at WP1.

\section{B. Measurements}

The first prediction from the tracking is the very existence of those bands. In Fig. 18 the time development of the horizontal half beam width is shown for varying initial beam sizes. The labels denote an initial inner scraper position after which it was retracted to $26 \mathrm{~mm}$ in all cases. In addition, the band border found in the simulation is shown as thick grey lines. The experimental data seem to be consistent with our phenomenological picture: the half beam width decreases in the outer band and increases in the lower band.

The second prediction is the attraction of particles towards lower amplitudes at WP1, which is clearly visible in Fig. 16(b). Owing to a large amplitude distribution this effect may, however, be screened by a considerable intensity loss as seen, for instance, in Fig. 12. It is therefore mandatory to scrape the beam tails so that the particles in the region of fast losses are removed but a sufficient number of particles remain in the amplitude band seen in parts (a) and (b) of Fig. 16. This type of experiment could be set up in the SPS and the result is shown in Fig. 19. The double-peak structure of a wire scan profile is shown right after the kick and 15 min later. The interesting feature is the shifting of the peaks down to smaller amplitudes without a reduction in peak height. This can only be interpreted as the predicted decrease of amplitude of a sizable fraction of the beam. This observation is not a singular event but was found in four separate experimental runs.

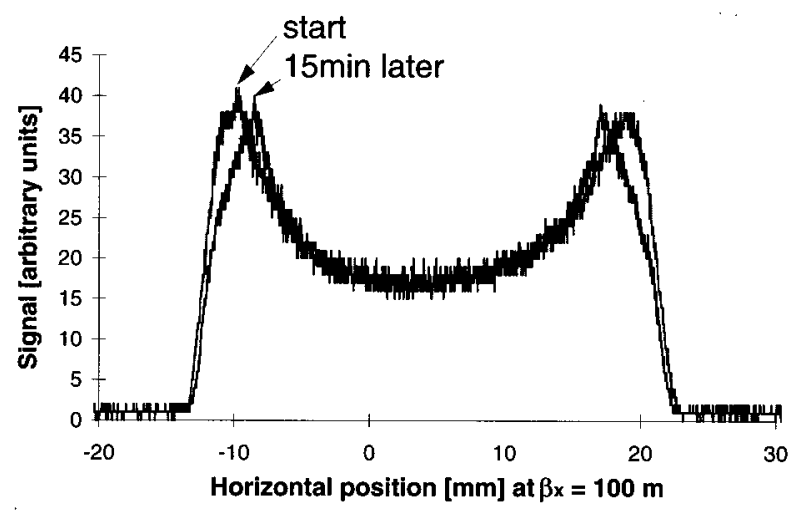

FIG. 19. Decreasing of the betatron amplitudes of a sizable fraction of the beam at WP1 (No. 65).

\section{CONCLUSIONS}

In 1993 we concluded the experiments intended to measure transport coefficients of particles in the chaotic regime by using scrapers to remove beam tails and limit the physical aperture. Despite a very careful preparation of the beam and the instrumentation it was not possible to obtain reproducible quantitative results. The qualitative results of earlier experiments could, however, be well reproduced: the loss rates increase with increasing tune modulation depth, and there is, contrary to the theoretical expectations, practically no dependence of the loss rates on the tune modulation frequency in the range from 9 to $180 \mathrm{~Hz}$. Two simultaneous modulation frequencies have a larger effect than a single one, and these latter experimental results are in qualitative agreement with theory.

In 1994 dynamic aperture measurements were done using the evolution of wire scan profiles. In the presence of strong tune modulation $\left(\Delta Q_{x}=1.87 \times 10^{-3}\right)$ the dynamic aperture could be reproduced with tracking simulation with a precision of $10 \%$. Without additional tune modulation this agreement deteriorates to some $20 \%$. We conclude that we still miss effects that are masked by the strong tune modulation.

In parallel, tracking simulations were performed with a view to extending our phenomenological understanding of the details of chaotic particle motion. An interesting outcome is the finding that the degree of chaos is not a good indicator for the survival time. There is also evidence from simulations and experiments that sizable fractions of particle distributions can move to smaller amplitudes. Moreover, a simple continuous diffusion model can be excluded for the description of the overall loss mechanism. Instead, a more complex model is needed that allows for sudden changes in the behavior of the particle motion when certain borders are reached in phase space.

\section{ACKNOWLEDGMENTS}

We wish to thank our colleagues from the accelerator physics group for participation in the experiment and discussions on the results, in particular J. Gareyte, W. Herr, J. Miles, and V. Ziemann. W. Scandale and T. Linnecar made an important contribution to the experiment in measuring the tune ripple spectrum. Essential to the success of the experiment was, of course, the help of the operations team: X. Altuna, C. Arimatea, R. Bailey, R. Billen, G. Buur, K. Cornelis, G. Crockford, B. Desforges, C. Despas, A. Faugier, A. Ferrari, R. Giachino, L. Normann, G. Robin, and A. Spinks. The data acquisition system was made operational and maintained by the following persons from the beam instrumentation group: A. Burns, H. Jakob, I. Milstead, and L. Vos. A data visualization program was provided by A. Sweeney and G. Morpurgo. The linear wire scanner system was adapted to our needs by J. Camas, G. Crockford, G. Ferioli, J. J. Gras, R. Jung, and J. Koopman. The kicker was kept operational and calibrated with the help of E. Carlier and L. Ducimetière. We thank M. Laffin for calibrating the scrapers and D. Bakker for technical support. Special thanks go to F. McIntosh for his continuous performance improvements of the computing facilities and programs. 
[1] H. Brück, R. Meinke, and P. Schmüser, Kerntechnik 56, 248 (1991).

[2] E. W. Collins et al. (unpublished); H. Brück et al., in Proceedings of the Second European Particle Accelerator Conference, Nice, 1990, edited by P. Marin and P. Mandrillon (Editions Frontières, Gif-sur-Yvette, 1990).

[3] T. Linnecar and W. Scandale, SPS Improvement Report 203, CERN SPS/DI-MST/TL/WS/EEK, 1986 (unpublished); L. Evans and J. Gareyte, in CERN Advanced Accelerator School, Oxford, 1985, edited by S. Turner (CERN, Geneva, 1987).

[4] J. Gareyte, Part. Accel. 27, 187 (1990).

[5] D. A. Edwards and M. J. Syphers, in Third U.S.-CERN School on Particle Accelerators, Anacapri, 1988, edited by M. Month and S. Turner, Lecture Notes in Physics Vol. 343 (Springer, Berlin, 1989), pp. 222-240.

[6] J. Gareyte, W. Scandale, and F. Schmidt, in International Workshop on Nonlinear Problems in Accelerator Physics, Berlin, 1992, edited by M. Berz, S. Martin, and H. Ziegler, IOP Conf. Proc. No. 131 (Institute of Physics, Bristol, 1993), pp. 235-248.

[7] O. Brüning et al., DESY Report No. HERA 95-05 and CERN Report No. CERN/SL/95-69 (AP), 1995 (unpublished).

[8] A. Chao et al., Phys. Rev. Lett. 61, 2752 (1988); S. G. Peggs, in Proceedings of the Second Advanced ICFA Beam Dynamics Workshop on Aperture-Related Limitations of the Performance and Beam Lifetime in Storage Rings, Lugano, 1988, edited by J. Hagel and E. Keil (CERN, Geneva, 1988); D. A. Edwards and M. J. Syphers, in Proceedings of the Second Advanced ICFA Beam Dynamics Workshop on Aperture-Related Limitations of the Performance and Beam Lifetime in Storage Rings, Lugano, 1988, edited by J. Hagel and E. Keil (CERN, Geneva, 1988), pp. 95-144; N. Merminga, Ph.D. thesis, University of Michigan, 1989 (unpublished); T. Chen et al., Phys. Rev. Lett. 68, 33 (1992); T. Satogata et al., ibid. 68, 1838 (1992); T. Satogata, Ph.D. thesis, Northwestern University, 1993 (unpublished).

[9] J. Y. Liu et al., Phys. Rev. E 49, 2347 (1994); Y. Wang et al., ibid. 49, 5697 (1994); M. Ellison et al., ibid. 50, 4051 (1994).

[10] S. Y. Lee, Proceedings of the International Workshop on Nonlinear Problems in Accelerator Physics (Ref. [6]).

[11] J. Bridge et al., Part. Accel. 28, 1 (1990); E. Crosbie et al., in Proceedings of the Particle Accelerator Conference, San Francisco, 1991, edited by L. Lizama and J. Chew (IEEE, New York, 1991); J. Liu et al., Part. Accel. 41, 1 (1993).

[12] P. Tran et al., in Nonlinear Dynamics in Particle Accelerators: Theory and Experiments, Italy, 1994, edited by S. Chattopadhyay, M. Cornacchia, and C. Pellegrini, AIP Conf. Proc. No. 344 (AIP, New York, 1995).

[13] R. L. Evans et al., CERN SPS/AMS/JG, SPS Improvement Report No. 209, 1986 (unpublished); A. Hilaire, in Proceedings of the Second Advanced ICFA Beam Dynamics Workshop on Aperture-Related Limitations of the Performance and Beam Lifetime in Storage Rings, Lugano, 1988, edited by J. Hagel and E. Keil (CERN, Geneva, 1988); L. R. Evans et al., in First European Conference on Particle Accelerators, Rome, 1988, edited by S. Tazzari (World Scientific, Singapore, 1989), pp. 619-621.
[14] K. Cornelis et al., CERN Report No. CERN SPS/88-45 (AMS), LHC Note 85; J. Gareyte, A. Hilaire, and F. Schmidt, in Proceedings of the Particle Accelerator Conference, Chicago, 1989, edited by F. Bennett and J. Kopta (IEEE, New York, 1989).

[15] D. Brandt et al., in Proceedings of the Second European Particle Accelerator Conference, Nice, 1990, edited by P. Marin and P. Mandrillon (Editions Frontières, Gif-sur-Yvette, 1990), pp. 1438-1440; X. Altuna et al., in Proceedings of the Advanced ICFA Beam Dynamics Workshop on Effects of Errors in Accelerators, their Diagnosis and Corrections, Corpus Christi, 1991, edited by A. W. Chao, AIP Conf. Proc. No. 255 (AIP, New York, 1992), pp. 355-369.

[16] M. Furman and F. Schmidt, Report No. CERN SPS/89-1 (AMS), SSCL-A-6.

[17] D. A. Edwards, F. Schmidt, and M. J. Syphers (private communication).

[18] O. Brüning, Part. Accel. 41, 131 (1993); Report No. DESY 94-085.

[19] A. Gerassimov, Report Nos. CERN SL/92-30 and FERMILAB-Pub-92/185.

[20] F. Galluccio and F. Schmidt, in Advanced ICFA Beam Dynamics Workshop on Effects of Errors in Accelerators, their Diagnosis and Corrections, Corpus Christi, 1991, edited by A. W. Chao, AIP Conf. Proc. No. 255 (AIP, New York, 1992), pp. 86-104.

[21] T. Linnecar and W. Scandale, in Proceedings of the 10th Particle Accelerator Conference, Santa Fe, 1983 [IEEE Trans. Nucl. Sci. NS30, Pt. 2 (1983)].

[22] A. Burns et al., in Conference Record of the 1993 IEEE Particle Accelerator Conference, Washington, DC, 1993, edited by S. T. Corneliussen (IEEE, New York, 1993), pp. 23012303.

[23] J. Bosser et al., Nucl. Instrum. Methods Phys. Res. A 235, 475 (1985).

[24] G. Ripken, Report No. DESY 85-084; F. Schmidt, Report No. CERN SL/94-56 (AP), found in WWW under http:// hpariel.cern.ch/frs/Documentation/doc.html.

[25] F. Willeke, in Proceedings of the Third Advanced Accelerator Physics Course, Uppsala, 1989, edited by S. Turner (CERN, Geneva, 1990); F. Schmidt, in Proceedings of the Workshop on Nonlinear Problems in Future Particle Accelerators, Capri, 1990, edited by W. Scandale and G. Turchetti (World Scientific, Singapore, 1991).

[26] F. Schmidt, Ph.D. thesis, Hamburg University (in German), Report No. DESY HERA 88-02; L. Schachinger and Y. Yan, Report No. SSC-N-664.

[27] A. Schoch, Report No. CERN 84-15.

[28] W. Fischer, Report Nos. DESY 95-235 and CERN SL/96-10 (AP).

[29] M. Seidel, Report No. DESY HERA 93-04.

[30] P. Burla et al., in Accelerator Physics at the Superconducting Super Collider, Dallas, TX, 1992-1993, edited by Y. T. Yan, J. P. Naples, and M. J. Syphers, AIP Conf. Proc. No. 326 (AIP, New York, 1995). 